\title{
Brief behavioral treatment for patients with treatment-resistant insomnia
}

\author{
This article was published in the following Dove Press journal: \\ Neuropsychiatric Disease and Treatment \\ 4 August 2016 \\ Number of times this article has been viewed
}

\section{Jihui Wang \\ Qinling Wei \\ Xiaoli Wu \\ Zhiyong Zhong \\ Guanying Li}

Department of Psychiatry, The Third Affiliated Hospital of Sun-Yat Sen University, Guangzhou, Guangdong, People's Republic of China
Correspondence: Jihui Wang Department of Psychiatry, The Third Affiliated Hospital of Sun-Yat Sen University, No 600, Tianhe Road, Tianhe District, Guangzhou, Guangdong 510630, People's Republic of China

Tel +86 I35 60I0 2194

Fax +862085252479

Email jonny928@I26.com
Objective: To evaluate the efficacy of brief behavioral treatment for insomnia (BBTI) in treating patients with treatment-resistant insomnia.

Methods: Seventy-nine adults with treatment-resistant insomnia were randomly assigned to receive either individualized BBTI (delivered in two in-person sessions and two telephone "booster" sessions, $n=40)$ or sleep hygiene education $(n=39)$. The primary outcome was subjective (sleep diary) measures of self-report symptoms and questionnaire measures of Pittsburgh sleep quality index (PSQI), insomnia severity index (ISI), Epworth sleeping scale (ESS), and dysfunctional beliefs and attitudes about sleep scale (DBAS).

Results: The repeated-measures analysis of variance showed significant time effects between pretreatment and posttreatment in the scale ratings of PSQI, ESS, DBAS, ISI, sleep latency (SL), time in bed (TIB), sleep efficiency (SE), and wake after sleep onset (WASO) in both groups and group $\times$ time interaction $\left(F_{\mathrm{PSQI}}=3.893, F_{\mathrm{ESS}}=4.500, F_{\mathrm{DBAS}}=5.530, F_{\mathrm{ISI}}=15.070, F_{\mathrm{SL}}=8.909\right.$, $F_{\mathrm{TIB}}=7.895, F_{\mathrm{SE}}=2.926$, and $\left.F_{\mathrm{WASO}}=2.595\right)$. The results indicated significant differences between BBTI and sleep hygiene in change scores of PSQI, ESS, DBAS, ISI, SL, TIB, SE, and WASO. Effect sizes were moderate to large.

Conclusion: BBTI is a simple and efficacious intervention for chronic insomnia in adults.

Keywords: brief behavioral treatment, treatment-resistant, insomnia

\section{Introduction}

Insomnia is the most prevalent sleep disorder that affects $6 \%-10 \%$ of adults. ${ }^{1}$ Cognitive behavioral therapy for insomnia (CBTI) is the first-line treatment option for insomnia, and there is strong empirical evidence of its efficacy. ${ }^{2-4}$ However, traditional CBTI has been encumbered by prolonged courses (which usually involve six to eight sessions), difficulty in acquiring, and shortage of professional therapists; thus, hypnotic medication is still a prevalent treatment in clinical practice, although it has several disadvantages such as poor long-term therapeutic effect, tolerance, addiction, and increase of fall-risk.

In order to enhance the clinical efficacy of CBTI, some researchers have developed brief behavioral treatment for insomnia (BBTI) in recent years, ${ }^{5}$ which mainly involves therapies of sleep restriction and stimulus control, which are thought to be the most efficacious behavioral techniques for primary insomnia. BBTI is found to have the following benefits: shortened period of treatment, which only involves about four sessions and one or two of them can be conducted over the telephone; brief contents, which lead to high patient compliance; no need for sophisticated training, which allows clinical nurses and junior therapists to take up the role; and focusing on "behavior", thus alleviating the stigma over "psychotherapy" in patients. ${ }^{5}$ Some studies have applied BBTI in the treatment of elderly patients with insomnia. Results showed that it has 
good clinical compliance and a significantly better therapeutic effect than sleep hygiene (SH) education. ${ }^{6}$ However, it has not been extensively reported internationally as to whether BBTI is applicable to populations of other age ranges and to patients with insomnia where previous hypnotic medication has showed repeated failures. This study focused on insomnia patients aged from 18 years to 65 years and compared the patient compliance and short-term therapeutic effect of BBTI versus $\mathrm{SH}$ education in an aim to provide more evidence for the wider application of BBTI.

\section{Methods}

\section{Study design}

This was a single-center randomized controlled trial with assessments at pretreatment and 4 weeks posttreatment. The primary outcome was subjective (sleep diary) measures of self-report symptoms and questionnaire measures of Pittsburgh sleep quality index (PSQI), insomnia severity index (ISI), Epworth sleeping scale (ESS), and dysfunctional beliefs and attitudes about sleep scale (DBAS). The ethics committee of Sun-Yat Sen University reviewed and approved the study protocol.

\section{Participants}

Participants were recruited from the outpatient clinics in the psychology department of the Third Affiliated Hospital of Sun-Yat Sen University between November 2013 and October 2014.

Patient volunteers were eligible for inclusion if they 1) were aged between 18 years and 65 years, 2) met research diagnostic criteria for insomnia disorder, which specify a sleep complaint lasting for at least 1 month, a mean total wake time (sleep onset + wake after onset) of $>60$ minutes per night during a screening week of sleep-diary monitoring, adequate opportunity and circumstances for sleep, and significant distress or daytime impairment, ${ }^{7} 3$ ) had at least two types of hypnotic medications in the past 6 months, each being administered continuously for at least 4 weeks, but insomnia still persists, 4) could gradually cease to use the hypnotic medications other than benzodiazepines (BZDs) 2 weeks before the study, and 5) provided written informed consent.

Exclusion criteria included dementia; epilepsy; schizophrenia, schizoaffective psychosis, bipolar disorder (antipsychotic may be used in such patients, and the sedative effects of those drugs probably alter the result); unstable somatic diseases; untreated other sleep disorders; idea and/or behavior of suicide recently; night shift; and experience of CBTI.
Individuals with treated depressive disorders, anxiety, or other sleep disorders were not excluded. Figure 1 illustrates participant flow.

\section{Procedure}

Potential subjects underwent a brief face-to-face screening, and those passing this stage completed structured interviews and sleep-diary monitoring for 1 week. Two clinical psychologists served as therapists and delivered BBTI and SH treatments. Another psychologist who was blind to the therapy conditions served as questionnaires assessor. All the questionnaires were assessed at pretreatment and 4 weeks posttreatment.

\section{Randomization}

Participants were randomly allocated to their respective treatment groups, using a randomization ratio of $1: 1$, by drawing lots. Allocation concealment was achieved by drawing cards from an opaque box. The questionnaires assessor and the assistants who recorded the data were blinded to group allocation.

\section{Treatments}

\section{Pharmacotherapy}

The participants who had been using antidepressants were required to keep their current dosages throughout the treatment. BZDs were not required to cease, but all the BZDs were changed to an equivalent daily dose of diazepam before the treatment. ${ }^{8,9}$ During the process of treatment, if the patients felt much better about the sleep quality, a reduction of $25 \%$ of the initial BZDs dose per week was considered.

\section{Brief behavioral treatment for insomnia}

BBTI is a 4-weekly manual-based intervention that included two in-person sessions on Weeks 1 and 3 and two telephone "booster" sessions on Weeks 2 and 4 (the detailed content is shown in supplementary materials). ${ }^{5}$

\section{Session I ( $\sim 60$ minutes)}

The first session aims at providing background information about SH recommendations, mechanisms of sleep regulation, theoretical basis of BBTI, and the following four chief contents of BBTI: 1) reduce time in bed (TIB); 2) get up at the same time every day; 3) do not go to bed unless sleepy; and 4) do not stay in bed unless asleep. Session 1 concludes with an individually tailored "sleep prescription" that includes recommended bedtime and wakeup time, activities to engage if not sleeping, medication plan if needed, and arrangements for follow-up sessions. 


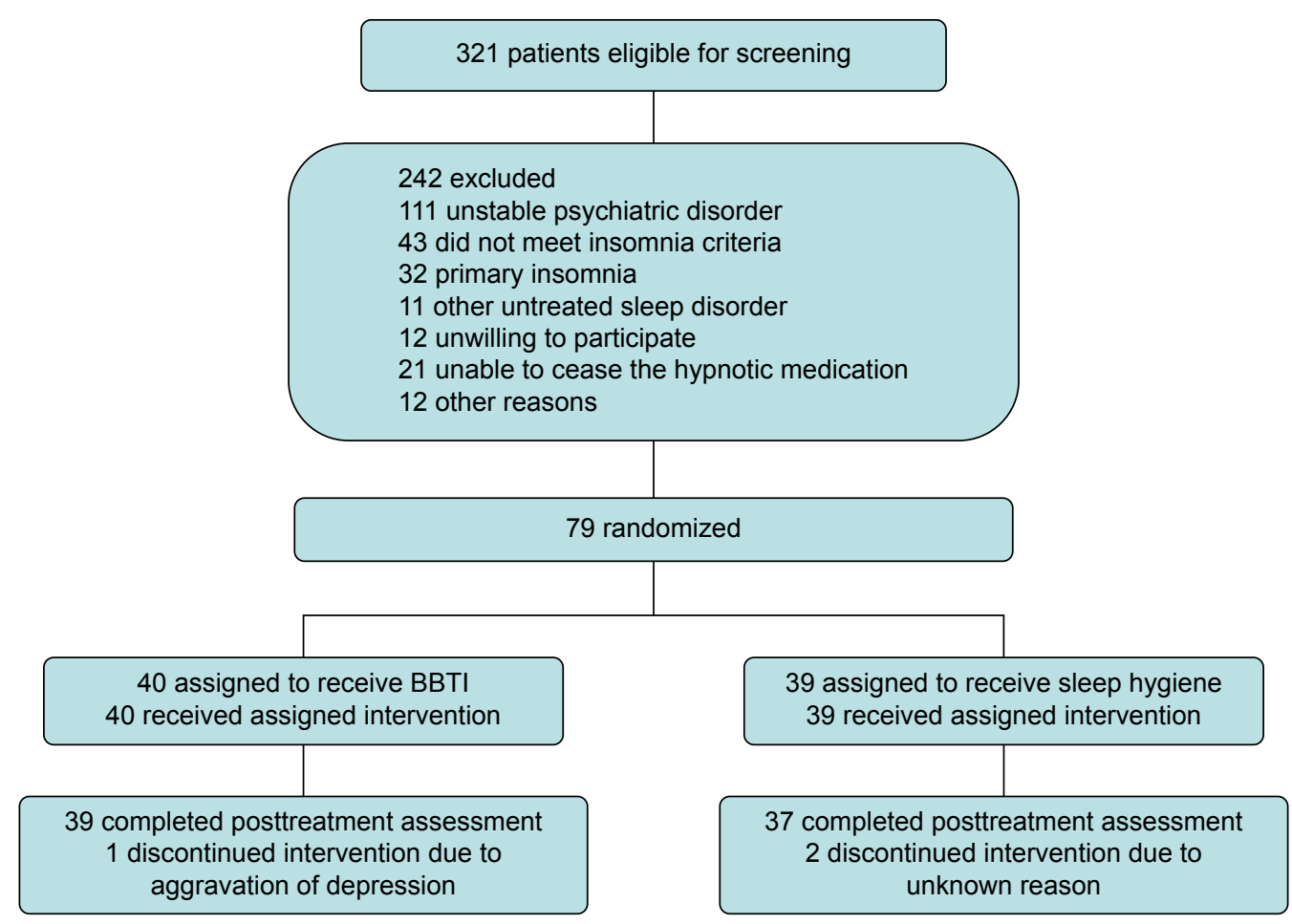

Figure I Study flowchart.

Abbreviation: BBTI, brief behavioral treatment for insomnia.

\section{Session 2 ( $\sim 5$ minutes)}

Session 2 is scheduled for a brief follow-up phone contact. Usually, the therapist asks general questions about the patient's sleep, daytime functioning, and treatment adherence. In other words, this phone call is used as a brief "check in" to provide support and to problem-solve issues regarding treatment adherence.

\section{Session 3 ( $\sim 30$ minutes)}

Session 3 is an in-person session intended to 1) review progress and difficulties occurring during the treatment, 2) monitor and reinforce adherence to treatment recommendations, and 3 ) guide the patient to regulate the sleep time according to the schedule.

\section{Session 4 ( $\sim 5$ minutes)}

Session 4 is the last follow-up phone contact that aims to review progress, resolve treatment-related difficulties, continue the regulation of sleep schedule, and discuss relapseprevention strategies.

\section{$\mathrm{SH}$ education}

Participants in the control group received four weekly treatment sessions (similar in length to those provided to the participants in the test group). In the initial session, the therapist presented the basic four $\mathrm{SH}$ recommendations, including avoiding caffeine or alcohol before bedtime, exercising regularly, avoiding being hungry or eating too much before bed, and maintaining a comfortable sleeping environment. During the next three sessions, the therapist reviewed the $\mathrm{SH}$ recommendations and engaged the participants in problem solving to address any treatment adherence problems.

\section{Measures}

\section{Sleep diary}

Each patient was required to complete a daily sleep diary. The variables extracted from the sleep diaries were TIB, total sleep time (TST), wake after sleep onset (WASO), sleep onset latency, sleep efficiency $(\mathrm{SE}=[\mathrm{TST} / \mathrm{TIB}] \times 100)$, and sleep quality (visual analog scale).

\section{Outcome questionnaires}

Participants were required to complete questionnaires on one occasion during the baseline period and a second time immediately following treatment.

\section{PSQI-Chinese version}

The PSQI-Chinese version is a 19-item questionnaire that has been demonstrated to have high internal consistency (0.85), test-retest reliability (0.83), and diagnostic validity. Scores from subscales are added to compute a global score of overall 
quality of sleep over the prior 1-week period. Global sleep quality scores are continuous (range 0-21) with high scores reflecting poor sleep quality. ${ }^{10}$

\section{Sleep severity index (ISI)}

The ISI consists of seven items that were used to measure insomnia severity. Total scores range from 0 to 28 , with high scores indicating greater insomnia severity. ${ }^{11}$

\section{Epworth sleepiness scale}

The ESS was used to measure the severity of daytime sleepiness. Respondents rated eight items regarding the likelihood of dozing in sedentary situations on a scale from 0 (never) to 3 (high chance). ${ }^{12}$

\section{Dysfunctional beliefs and attitudes about sleep scale} The DBAS consists of 30 items that measure sleep-related beliefs or attitudes. The total score of DBAS is calculated from the average score of all the items and ranges from 0 to 10 with high scores indicating more dysfunctional beliefs. ${ }^{13}$

\section{Statistical methods}

The results were statistically analyzed using SPSS 16.0. Participant characteristics were compared using Mann-Whitney $U$-test and $\chi^{2}$ tests. Continuous variables (general sleep measures and sleep diary) were evaluated by repeated-measures analyses of variance (ANOVA). Factors were group (BBTI vs $\mathrm{SH})$, time (pretreatment or posttreatment), and group $\times$ time interaction. Drop-out cases were taken into account for the statistical analysis. Their data were estimated through last-observation-carried-forward analysis. Significance level was set at $\alpha=0.05$, through two-sided test.

\section{Results}

\section{General results}

Of the 79 participants originally enrolled in the study, 76 completed the 4-week treatment protocol (43 women, 36 men). Mean age was $41.16 \pm 8.42$ years, and the duration of insomnia was on average $4.67 \pm 2.32$ years. Sociodemographic and clinical characteristics are shown in Table 1. Treatment group and control group did not differ significantly.

\section{Comparisons of general sleep measures}

The repeated-measures ANOVA for general sleep measures showed significant time effects between pretreatment and posttreatment in the scale ratings of PSQI, ESS, DBAS, and ISI in both groups $(P<0.05)$ and group $\times$ time interaction $\left(F_{\mathrm{PSQI}}=3.893, P=0.005 ; F_{\mathrm{ESS}}=4.500, P=0.002 ; F_{\mathrm{DBAS}}=5.530\right.$, $\left.P<0.001 ; F_{\text {ISI }}=15.070, P<0.001\right)$. The results indicated significant differences between BBTI and $\mathrm{SH}$ in improvements of PSQI, ESS, DBAS, and ISI. Effect sizes were moderate to large (Table 2).

Table I Demographic materials and clinical characteristics at baseline

\begin{tabular}{|c|c|c|c|c|}
\hline \multirow[t]{2}{*}{ Variables } & \multirow{2}{*}{$\frac{\text { Test group }}{(n=40)}$} & \multirow{2}{*}{$\frac{\text { Control group }}{(n=39)}$} & \multirow[t]{2}{*}{$Z \mid \chi^{2}$} & \multirow[t]{2}{*}{$P$-value } \\
\hline & & & & \\
\hline Age, years, mean (SD) & $42.70(8.83)$ & $39.63(7.84)$ & $Z=1.296$ & 0.192 \\
\hline Sex, n & & & $\chi^{2}=0.011$ & 0.918 \\
\hline Male & 18 & 18 & & \\
\hline Female & 22 & 21 & & \\
\hline Marital status, $\mathrm{n}$ & & & $\chi^{2}=0.692$ & 0.406 \\
\hline Married & 31 & 27 & & \\
\hline Other & 9 & 12 & & \\
\hline Employment status, $\mathrm{n}$ & & & $\chi^{2}=0.307$ & 0.58 \\
\hline Employed & 27 & 24 & & \\
\hline Other & 13 & 15 & & \\
\hline With other mental comorbidity, $\mathrm{n}$ & & & $\chi^{2}=0.728$ & 0.394 \\
\hline Yes & 8 & 11 & & \\
\hline No & 32 & 28 & & \\
\hline With other medical comorbidity, $\mathrm{n}$ & & & $\chi^{2}=0.837$ & 0.36 \\
\hline Yes & 6 & 9 & & \\
\hline No & 34 & 30 & & \\
\hline Duration of insomnia, years, mean (SD) & $4.77(2.37)$ & $4.57(2.30)$ & $Z=0.391$ & 0.694 \\
\hline Duration of using BZDs, years, mean (SD) & $16.67(9.53)$ & $17.53(10.07)$ & $Z=0.334$ & 0.736 \\
\hline Dosage of BZDs, mg/d, mean (SD) & II.53 (6.98) & $10.78(7.24)$ & $Z=0.636$ & 0.522 \\
\hline
\end{tabular}

Abbreviation: BZDs, benzodiazepines. 
Table 2 General sleep measures

\begin{tabular}{|c|c|c|c|c|c|}
\hline \multirow[t]{2}{*}{ Measures } & \multicolumn{2}{|l|}{ BBTI $(n=40)$} & \multicolumn{2}{|l|}{ SH $(n=39)$} & \multirow{2}{*}{$\begin{array}{l}\text { BBTI-SH } \\
\text { difference (SEM) } \\
\text { [95\% } \mathrm{Cl}], \text { Cohen's } \\
\text { effect size }\end{array}$} \\
\hline & $\begin{array}{l}\text { Pretreatment/ } \\
\text { posttreatment, } \\
\text { mean (SD) }\end{array}$ & $\begin{array}{l}\text { Difference (SEM) } \\
{[95 \% \mathrm{CI}]}\end{array}$ & $\begin{array}{l}\text { Pretreatment/ } \\
\text { posttreatment, } \\
\text { mean (SD) }\end{array}$ & $\begin{array}{l}\text { Difference (SEM) } \\
{[95 \% \mathrm{Cl}]}\end{array}$ & \\
\hline PSQI & $10.43(2.78) / 5.50(1.27)$ & $4.63(0.42)$ [3.78-5.48] & $10.13(2.93) / 7.16(1.62)$ & $2.96(0.37)$ [2.19-3.74] & $\begin{array}{l}\mathrm{I} .66(0.6 \mathrm{I})[0.40-2.93] \\
0.74\end{array}$ \\
\hline ESS & $5.93(4.78) / 2.36(2.60)$ & $3.56(0.59)$ [2.36-4.77] & $5.86(4.28) / 5.03(3.44)$ & $1.66(0.43)[0.77-2.56]$ & $\begin{array}{l}1.90(0.58)[0.70-3.90] \\
0.69\end{array}$ \\
\hline DBAS & $5.90(0.62) / 4.88(0.89)$ & $1.02(0.10)[0.81-1.23]$ & $5.59(0.74) / 5.09(0.99)$ & $0.49(0.13)[0.22-0.77]$ & $\begin{array}{l}0.52(0.18)[0.14-0.90] \\
0.8\end{array}$ \\
\hline ISI & I2.93 (2.89)/7.96 (1.99) & $4.77(0.60)[3.53-6.00]$ & $12.00(3.12) / I 1.36(2.52)$ & $2.63(0.47)$ [1.66-3.60] & $\begin{array}{l}2.13(0.74)[0.61-3.64] \\
0.86\end{array}$ \\
\hline
\end{tabular}

Abbreviations: $\mathrm{BBTI}$, brief behavioral treatment for insomnia; Cl, confidence interval; SH, sleep hygiene; PSQI, Pittsburgh sleep quality index; ESS, Epworth sleepiness scale; DBAS, dysfunctional beliefs and attitudes about sleep scale; ISI, insomnia severity index; SEM, standard error of the mean.

\section{Comparisons of sleep diary}

The repeated-measures ANOVA for sleep diary measures showed a significant time effects between pretreatment and posttreatment in the scale ratings of sleep latency (SL), TIB, SE, WASO, and sleep quality in both groups $(P<0.05)$ and group $\times$ time interaction $\left(F_{\mathrm{SL}}=8.909, P<0.001 ; F_{\mathrm{TIB}}=7.895\right.$, $P<0.001 ; F_{\mathrm{SE}}=2.926, P=0.024 ; F_{\mathrm{WASO}}=2.595, P=0.046$; $\left.F_{\text {sleep quality }}=6.457, P<0.001\right)$. The results indicated significant differences between BBTI and SH in the improvements of SL, TIB, SE, WASO, and sleep quality. Effect sizes were moderate to large (Table 3).

\section{Use of BZDs}

There were eight cases in the test group (26.7\%) and two cases in the control group (6.7\%) who stopped using BZDs.
The difference has statistical significance $\left(\chi^{2}=4.320, P<0.05\right)$. The average nightly dose of BZDs in the test group decreased from $11.54 \mathrm{mg} / \mathrm{d}$ at baseline to $6.42 \mathrm{mg} / \mathrm{d}$ at posttreatment, the difference was $5.12 \mathrm{mg} / \mathrm{d}$. The average nightly dose of BZDs in the control group decreased from $10.78 \mathrm{mg} / \mathrm{d}$ at baseline to $9.68 \mathrm{mg} / \mathrm{d}$ at posttreatment, the difference was $1.10 \mathrm{mg} / \mathrm{d}$. There was a statistical significance in the differences of BZDs between groups and within groups $(Z=4.803, P<0.01)$.

\section{Discussion}

SH education was thought to be one of the basic intervention measures of insomnia. Our research shows that for patients with treatment-resistant insomnia between 18 years and 65 years old, 4 weeks of BBTI treatment could effectively improve the sleep quality and daytime functions.

Table 3 Sleep diary measures

\begin{tabular}{|c|c|c|c|c|c|}
\hline \multirow[t]{2}{*}{ Measures } & \multicolumn{2}{|l|}{ BBTI $(n=40)$} & \multicolumn{2}{|l|}{ SH (n=39) } & \multirow{2}{*}{$\begin{array}{l}\text { BBTI-SH } \\
\text { difference (SEM) [95\% CI], } \\
\text { Cohen's d effect size }\end{array}$} \\
\hline & $\begin{array}{l}\text { Pretreatment/ } \\
\text { posttreatment, } \\
\text { mean (SD) }\end{array}$ & $\begin{array}{l}\text { Difference (SEM) } \\
{[95 \% \mathrm{CI}]}\end{array}$ & $\begin{array}{l}\text { Pretreatment/ } \\
\text { posttreatment, } \\
\text { mean (SD) }\end{array}$ & $\begin{array}{l}\text { Difference (SEM) } \\
{[95 \% \mathrm{Cl}]}\end{array}$ & \\
\hline \multirow[t]{2}{*}{ SL } & $35.06(17.32) / 18.10(8.46)$ & $15.83(2.13)$ & $34.40(15.33) / 29.70(13.04)$ & $8.93(1.66)$ & $6.90(3.09)[0.56-13.23]$ \\
\hline & & [16.47-20.19] & & {$[5.53-12.33]$} & 0.68 \\
\hline \multirow[t]{2}{*}{ TST } & $350.20(61.40) / 356.97(38.84)$ & $-6.77(10.22)$ & $343.23(65.10) / 361.10(61.49)$ & $-17.56(5.93)$ & $11.10(11.84)[-13.11$ to 35.31$]$ \\
\hline & & {$[-27.72$ to $\mid 4.19]$} & & {$[-30.00$ to -5.72$]$} & 0.24 \\
\hline \multirow[t]{2}{*}{ TIB } & $54 I .42(92.95) / 444.04$ (56.57) & $97.38(13.32)$ & $536.60(118.59) / 511.54$ & $25.05(9.89)$ & 72.32 (I5.47) [40.46-I03.98] \\
\hline & & {$[70.15-124.62]$} & $(95.07)$ & {$[4.8 \mathrm{I}-45.29]$} & 1.13 \\
\hline \multirow[t]{2}{*}{ SE } & $0.65(0.16) / 0.81(0.10)$ & $-0.15(0.03)[-0.20$ & $0.66(0.16) / 0.72(0.14)$ & $-0.05(0.14)[-0.08$ & $-0.08(0.02)[-0.14$ to -0.02$]$ \\
\hline & & to -0.09$]$ & & to -0.02$]$ & 0.76 \\
\hline \multirow[t]{2}{*}{ WASO } & $156.15(116.49) / 68.96(55.12)$ & $88.32(18.88)$ & 158.96 (I28.98)/I20.74 & $33.98(11.25)$ & $54.32(21.21)$ [10.93-97.72] \\
\hline & & [49.70-126.93] & $(99.11)$ & {$[10.97-57.00]$} & 0.64 \\
\hline \multirow[t]{2}{*}{ SQ, 0-100 } & $54.32(5.55) / 66.89(6.76)$ & $12.57(4.03)$ & $52.98(6.01) / 56.77(6.94)$ & $3.79(3.22)[-2.51$ & $8.78(5.63)[2.66-14.90]$ \\
\hline & & {$[6.80-19.37]$} & & to 9.07$]$ & 0.83 \\
\hline
\end{tabular}

Abbreviations: BBTI, brief behavioral treatment for insomnia; Cl, confidence interval; SH, sleep hygiene; SL, sleep latency; TST, total sleep time; TIB, time in bed; SE, sleep efficiency; WASO, wake after sleep onset; SQ, sleep quality; SEM, standard error of the mean. 
The therapeutic effects of BBTI are significantly better than SH education alone.

Due to the differences of study design, it is somewhat difficult to carry out comparisons among the therapeutic results of different components of behavioral treatment. Two recent review papers suggest that, whether it is for primary or comorbid insomnia, CBTI could effectively ameliorate the sleep qualities of patients with chronic insomnia, as well as enhance their daytime functions, with an effect size of "moderate to significant", however most of these studies have adopted traditional CBTI therapy. ${ }^{4,14}$ Buysse et $\mathrm{al}^{6}$ have carried out 4 weeks of BBTI to elderly patients, and results showed that BBTI, similar to CBTI, could also effectively ameliorate the sleep qualities of patients with chronic insomnia. Our research also shows that the scale ratings of ISI and PSQI in the test group are significantly lowered, suggesting an enhancement of general sleep quality. Reviewing of sleep diary suggests that the enhancements of SL, SE, WASO, and sleep quality in the test group are all better than those in the control group, with an effect size of $0.68,0.76,0.64$, and 0.83 , respectively. Insomnia is regarded as a 24-hour disease because daytime function of the patients is as important as sleep complaints during nighttime. In this study, the participants in the test group made more improvement than those in the control group on ESS scores, which reflect the daytime function of sleepiness. These results suggest that, to patients with insomnia where the therapeutic effect of hypnotic medication has proved to be poor, 4 weeks of BBTI therapy could achieve better effect than SH education. Furthermore, the effects of BBTI may not be contributed by pharmacotherapy effect because the participants of the test group make more progress during the behavior treatment than those of the control group in reducing BZDs use, which is one of the targets of the behavior treatment.

In addition, a large reduction was observed in TIB in the test group versus control group, with an effect size of "large". "Reducing TIB" is one of the core components of BBTI; the patient is in compliance with the requirement of BBTI and actively willing to shorten the TIB. Also, the reduction of dosage of hypnotics could be another reason. ${ }^{15}$ Further research targeting in drug-free patients might be helpful for analysing the cause-effect relationship.

Patients with insomnia usually have cognitive misconceptions about sleep and its daytime effects, which eventually lead to increased arousal and persistence of their insomnia. ${ }^{16}$ Traditional CBTI usually has content of cognitive restructuring to rectify the cognitive misconceptions of the patients. ${ }^{17}$
Different from traditional methods, BBTI has focused more on the "behavioral changes" of the patients. However, the results of our research suggest that the scale rating of DBAS in the test group is significantly better than the control group, with an effect size of 0.73 , suggesting that even the simplest form of behavioral therapy could in a sense ameliorate the cognitive misconceptions of the patients, which is challenged by the actual effect of the behavioral therapy. For instance, patients with insomnia usually have cognitive misconceptions that "sleep time could be increased through the increase of TIB", ${ }^{18}$ whereas behavioral therapy asks the patients to "reduce the TIB in order to enhance sleep quality". If the behavioral therapy proves effective, the cognitive misconceptions of the patients are naturally challenged and then changed. Thus, for those patients who are stubborn in changing their cognitive misconceptions in the early stage of CBTI, maybe it is better to apply cognitive therapy when behavioral therapy has achieved certain effects, because at that time it is easier for them to realize their cognitive misconceptions and be willing to make change.

\section{Limitations}

Our study includes certain notable limitations. First, the observation periods are relatively short without follow-up study of the long-term prognosis of the therapy; second, we did not exclude the participants taking hypnotic medications, thus the influence of medication to the research results should not be neglected; third, there are no objective measures, for example, pre- and posttreatment polysomnography. Considering the fact that hypnotic medications are very common in the real-world treatment of comorbid insomnia, the medications used between the two groups were basically similar on baseline, and the participants of the test group make more progress during the behavior treatment than those of the control group in reducing BZDs use, the effects of BBTI may not be contributed by pharmacotherapy effect. As far as the objective measures are concerned, self-reported feelings are as important as objective measures in patients with insomnia, and self-reported measures are more commonly used in routine work, so this study has some clinical significance in real-life practice.

\section{Conclusion}

BBTI produced meaningful improvements for adult patients with treatment-resistant insomnia in actual practice settings. Patients tolerated the treatment well. We suggest future studies examine the effectiveness of BBTI in a longer period. 


\section{Acknowledgments}

This study was supported by three grants: Medical research foundation of Guangdong Province, grant No A2015125, Science and Technology Planning Project of Guangdong Province, grant No 2013B021800085 and the Fundamental Research Funds for the Central University, grant No 14ykpy28. The funding institutions had no role in study design, data collection and analysis, decision to publish, or preparation of the manuscript. No additional external funding was given to the study. The authors would like to thank the staff at the Third Affiliated Hospital of Sun-Yat Sen University for their assistance with this research.

\section{Disclosure}

The authors report no conflicts of interest in this work.

\section{References}

1. Ohayon MM, Reynolds CF 3rd. Epidemiological and clinical relevance of insomnia diagnosis algorithms according to the DSM-IV and the international classification of sleep disorders (ICSD). Sleep Med. 2009; 10(9):952-960.

2. Morin CM, Benca R. Chronic insomnia. Lancet. 2012;379(9821): 1129-1141.

3. Mitchell MD, Gehrman P, Perlis M, Umscheid CA. Comparative effectiveness of cognitive behavioral therapy for insomnia: a systematic review. BMC Fam Pract. 2012;13:40.

4. Okajima I, Komada Y, Inoue Y. A meta-analysis on the treatment effectiveness of cognitive behavioral therapy for primary insomnia. Sleep Biol Rhythms. 2011;9(1):24-34.

5. Troxel WM, Germain A, Buysse DJ. Clinical management of insomnia with brief behavioral treatment (BBTI). Behav Sleep Med. 2012;10(4): 266-279.
6. Buysse DJ, Germain A, Moul DE, et al. Efficacy of brief behavioral treatment for chronic insomnia in older adults. Arch Intern Med. 2011;171(10):887-895.

7. Sleep Disorder Research Group of the Neurology Chapter of Chinese Medical Association. Guidelines of diagnosis and treatment of insomnia in adult Chinese population. Zhonghua Shen Jing Ge Za Zhi. 2012;45: 534-540.

8. Valenstein M, Taylor KK, Austin K, Kales HC, McCarthy JF, Blow FC. Benzodiazepine use among depressed patients treated in mental health settings. Am J Psychiatry. 2004;161(4):654-661.

9. Zitman FG, Couvée JE. Chronic benzodiazepine use in general practice patients with depression: an evaluation of controlled treatment and taper-off: report on behalf of the Dutch Chronic Benzodiazepine Working Group. Br J Psychiatry. 2001;178:317-324.

10. Liu X, Tang M, Hu L, et al. Reliability and validity of the Pittsburgh sleep quality index. Chin J Psychiatry. 1996;29(2):103-107.

11. Bastien $\mathrm{CH}$, Vallières A, Morin CM. Validation of the insomnia severity index as an outcome measure for insomnia research. Sleep Med. 2001, 2(4):297-307.

12. Johns MW. A new method for measuring daytime sleepiness: the Epworth sleepiness scale. Sleep. 1991;14(6):540-545.

13. Espie CA, Inglis SJ, Harvey L, Tessier S. Insomniacs' attributions. Psychometric properties of the dysfunctional beliefs and attitudes about sleep scale and the sleep disturbance questionnaire. J Psychosom Res. 2000;48(2):141-148.

14. Sánchez-Ortuño MM, Edinger JD. Cognitive-behavioral therapy for the management of insomnia comorbid with mental disorders. Curr Psychiatry Rep. 2012;14(5):519-528.

15. Lader M. Effectiveness of benzodiazepines: do they work or not? Expert Rev Neurother. 2008;8(8):1189-1191.

16. Harvey AG. A cognitive model of insomnia. Behav Res Ther. 2002; 40(8):869-893.

17. Pigeon WR. Treatment of adult insomnia with cognitive-behavioral therapy. J Clin Psychol. 2010;66(11):1148-1160.

18. Buysse DJ, Germain A, Hall M, Monk TH, Nofzinger EA. A neurobiological model of insomnia. Drug Discov Today Dis Models. 2011;8(4): $129-137$. 


\section{Supplementary materials Session content for BBTI' \\ Session I}

The first session aims at providing background information about sleep hygiene recommendations, mechanisms of sleep regulation, and the theoretical basis of brief behavioral treatment for insomnia (BBTI) therapy. A simple manual was used by the therapist to work through with the patient in the sessions. Using the manual, the therapist begins with a brief review of behaviors that "hurt" or "help" sleep and then explains to the patient that there are two basic processes that control sleep. The first process is "sleep drive". Sleep drive can be thought of as a rubber band - the longer one has been awake, the more stretched the band becomes. By the end of the day, if the patient has been awake the whole daytime, that rubber band should be really tight, so that it would be much easier for the patient to fall asleep deeply and quickly. It is like the rubber band bouncing back. The second factor that regulates sleep is the body's internal biological clock that is "set" to promote sleep at night and wakefulness in the daytime. This process controls the timing of when we are likely to sleep optimally and function at our best during the day. Ideally, these two processes that regulate our sleep work together. In brief, sleep drive by the end of the day caused by the long time state of being awake helps the patient to fall asleep quickly, while the biological clock helps him or her to stay asleep later in the night.

After discussing the treatment rationale, the therapist reviews the patient's sleep diaries and works with the patient to calculate average sleep parameters in the past week (ie, bedtime, wake time, sleep latency, wakefulness after sleep onset, total time in bed [TIB], and total sleep time [TST]). This process often helps the patient to recognize potential sleep-interfering behaviors (eg, total TIB is much longer than TST).

Next, the therapist guides the patient to use the four basic "rules" to improve sleep. It is critical to keep in mind that the rules should be set individually and specifically.

\section{Rule I}

Reduce the TIB. TIB should be limited to match the actual sleep time +30 minutes. This recommendation might be more acceptable for the patient if the therapist acknowledged that it was challenging at first and then explained to the patient its importance to the homeostatic sleep drive.

\section{Rule 2}

Wake up at the same time of day every day. Patients with insomnia often have the tendency to "sleep in" on weekends or try to "catch up" on sleep after a poor night of sleep. This behavior actually perpetuates the insomnia problem by reducing both the biological clock's signal for sleep and wakefulness, as well as the homeostatic drive for sleep on the next night.

\section{Rule 3}

Do not go to bed unless you are sleepy. It should be emphasized that "trying" to sleep is not only frustrating, but biologically impossible: sleep is not an intended behavior, but a state that the brain switches into when it is ready to do so, based on homeostatic and circadian factors. After reviewing the patient's sleep diaries and interviewing the patient, the therapist should have a good understanding and try to change the patient's typical belief that they "should" be going to bed to achieve better sleep even when they do not have any sensation of sleepiness.

\section{Rule 4}

Do not stay in bed unless you are asleep. According to the principle of stimulus control, it is believed that through continuous nights of being unable to sleep in bed, the brain develops a learned association between the bed and being awake, rather than being asleep. In other words, insomnia is partly a "learned habit" of staying awake in bed. Furthermore, staying in bed perpetuates the round of wakefulness-frustrationarousal-wakefulness. To break this habit, the patient is required to get out of bed if awake for $>30$ minutes (by their own estimate). In addition, to successfully carry out this recommendation, it is critical that the therapist brainstorm with the patient individualized and specific activities that he or she can do if not sleeping. These activities should be distracting. The activities which are overly stimulating such as work-related activities or using computer should be avoided. This recommendation aims to distract the patients from attention to the fact that he or she is not sleeping. The patient is supposed to repeat the activity until he or she feels sleepy enough to return to bed.

\section{Setting the prescribed sleep schedule}

Session 1 concludes with an individually tailored "sleep prescription" that includes recommended bedtime and wakeup time, activities to engage in if not sleeping, medication plan if needed, and making arrangements for follow-up sessions. Usually, it is helpful to set the wakeup time first and then decide the bedtime based on total prescribed TIB. Finally, it is necessary to remind patients that BBTI, like other behavioral treatments, may have side effects (eg, daytime sleepiness and 
fatigue). Therefore, some safety issues (eg, sleepiness during long-distance driving) are also discussed with patients. Meanwhile, directly acknowledging the difficulty in implementing the new schedule is helpful, not only in setting realistic expectations about the course and outcome of BBTI, but also in enhancing treatment adherence.

\section{Session 2 (phone contact)}

Session 2 is scheduled for a brief follow-up review through phone contact. During the phone call, the patient is required to prepare the sleep diary in hand for the review of the past week. Usually, the therapist asks general questions about the patient's sleep, daytime functioning, and treatment adherence. In other words, this phone call is used as a brief "check in" to provide support and to problem solve issues regarding treatment adherence.

\section{Session 3}

Week 3 is an in-person session intended to 1) review progress and difficulties occurring during the treatment, 2) monitor and reinforce adherence to treatment recommendations, and 3) guide the patient to regulate the sleep time according to the schedule. Specifically, if sleep onset latency and wake (time) after sleep onset are $<30$ minutes on most nights according to the patient's sleep diaries, then 15 minutes is added to the TST. The patient is supposed to maintain this new sleep schedule for the next week. If, on the other hand, sleep onset latency or wake after sleep onset are $>30$ minutes, then TIB should be decreased by 15 minutes. It is important to explain to the patient that the therapist is not there to criticize him or her but rather to achieve an expected sleep schedule through a trial and error process. Finally, in this session, it is also helpful to motivate the patient to overcome initial difficulties because progress always comes with repeated practice.

\section{Session 4}

Session 4 is the last follow-up phone contact that aims to review progress, resolve treatment-related difficulties, continue the regulation of sleep time according to the schedule, and discuss relapse-prevention strategies. Particularly, the last session is used to 1) review the instructions for stimulus control and sleep restriction, 2) make sure that the patient understands the titration principle of sleep schedule, and 3) discuss with the patient possible sleep disturbances that they may encounter in the future. Thinking ahead to these situations may help the patient to develop proactive strategies for managing these problems.

\section{Reference}

1. Troxel WM, Germain A, Buysse DJ. Clinical management of insomnia with brief behavioral treatment (BBTI). Behav Sleep Med. 2012;10:266-279.
Neuropsychiatric Disease and Treatment

\section{Publish your work in this journal}

Neuropsychiatric Disease and Treatment is an international, peerreviewed journal of clinical therapeutics and pharmacology focusing on concise rapid reporting of clinical or pre-clinical studies on a range of neuropsychiatric and neurological disorders. This journal is indexed on PubMed Central, the 'PsycINFO' database and CAS

\section{Dovepress}

and is the official journal of The International Neuropsychiatric Association (INA). The manuscript management system is completely online and includes a very quick and fair peer-review system, which is all easy to use. Visit http://www.dovepress.com/testimonials.php to read real quotes from published authors. 